\title{
Modeling Neurons of the Inferior Colliculus
}

\author{
Harry Erwin ${ }^{1}$, Mark Elshaw ${ }^{1}$, Adrian Rees ${ }^{2}$, David Perez-Gonzalez ${ }^{2}$, and \\ Stefan Wermter ${ }^{1}$ \\ ${ }^{1}$ School of Computing and Technology, University of Sunderland, SR6 0DD, \\ United Kingdom \\ ${ }^{2}$ Institute of Neuroscience, The Medical School, \\ Newcastle University, NE2 4HH, United Kingdom \\ Harry.Erwin@sunderland.ac.uk, Mark.Elshaw@sunderland.ac.uk, \\ Adrian.Rees@newcastle.ac.uk, David.Perez-Gonzalez@newcastle.ac.uk, \\ Stefan.Wermter@sunderland.ac.uk
}

\begin{abstract}
The MiCRAM project has been developing detailed neural models of the inferior colliculus (IC) as the basis for understanding the neural networks that underlie early auditory processing at that level. The initial phase of this project has been to develop biologically plausible models of the physiologically distinct cell types of the IC. This has led to the recognition that some of the gross behavior that produces delay sensitivity may be the result of detailed dynamic interactions between multiple channels.
\end{abstract}

Keywords: Neural modeling, neurodynamics, audition.

\section{Introduction}

The MiCRAM project is a collaborative study involving the Universities of Sunderland and Newcastle in developing a biologically plausible model of auditory processing at the level of the inferior colliculus (IC). This is being done to clarify the roles of the spectral and temporal representations in the IC and investigate how they interact with auditory processing to focus attention and select sound sources for analysis.

The IC is organized tonotopically into parallel frequency lamina, each with a collection of disc and stellate cells with a variety of response patterns to pure tone stimuli [1]. The IC might be seen as a large set of full and partial spectral representations. Sound sources at different locations or with different characteristics activate the IC spectral representations differently for different sound sources. Understanding how this takes place will provide insight into the auditory processes in acoustically cluttered environments.

The first step towards this goal is the development of biologically realistic GENESIS [2] models of individual IC. That requires us to explore statistically valid models of the firing patterns of these cells.

A number of research groups are doing research into the area of statistically valid cell models [3-5]. Since spike trains are binary in form, evaluating goodness of fit is more difficult than for continuous processes [6]. Although IC disk cells fire in a 
reliable way to repeated presentations of the same stimulus, they often respond quite differently if the intensity or structure of the stimulus is changed, which makes it difficult to use quantitative models to classify these cells [1].

The PSTH classifications in Rees, et al [1] emphasize the spiking rate modulation of IC cells in response to pure tone stimuli in vivo and so reflect the cell's membership in neural networks. This can be seen most clearly in pauser cells that spike a variable number of times initially, then become quiet for a period, and finally spike continuously or at a modulated rate (Figure 1). Afterhyperpolarisation can produce a similar but distinct pattern (Figure 2), that is more regular with a single spike reliably appearing in each burst.

\section{Pauser cell in vivo}

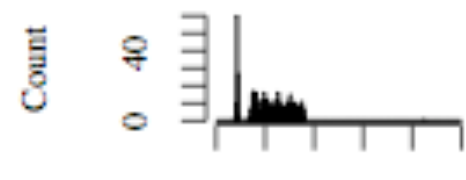

$0100 \quad 200$

\section{Simulated pauser cell}

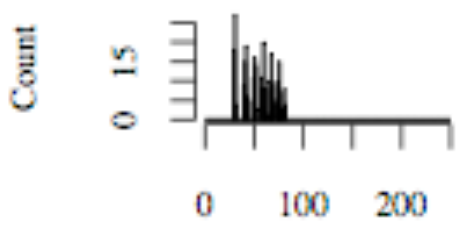

Time (msec)
Figure 1 Pauser cell PTSH, 100 in vivo trials, pure tone stimulus lasting $75 \mathrm{msec}$, one $\mathrm{msec}$ bins (see [1]).
Figure 2, PTSH showing effect of afterhyperpolarisation in simulated pauser cell.

The contribution of specific membrane conductances to the generation of these response patterns has been reported by Sivaramakrishnan and Oliver [7]. The MiCRAM program is modeling these cell types to explore their dynamics and incorporate them into large scale network models of the IC.

\section{Methods}

The MiCRAM program models these cell types using the GENESIS neural modeling tool. The channel properties are based on the models in [8]. The simulations replicate their experiments, with a medium goal of constructing networks of different classes of these cells. 


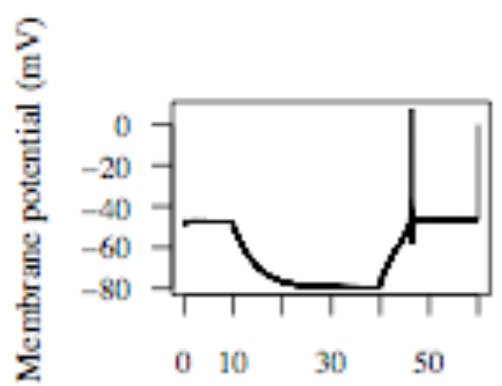

Time (msec)

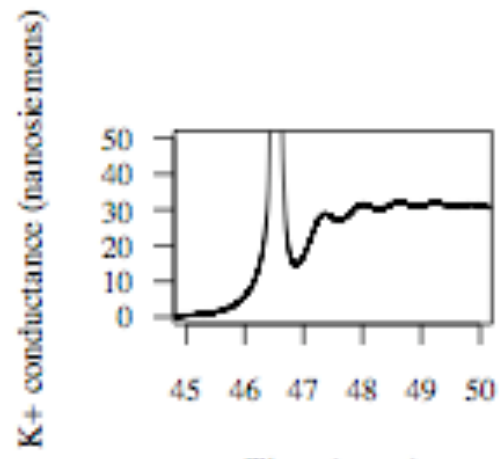

Time (msec)

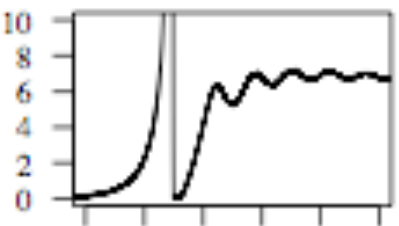

$\begin{array}{llllll}45 & 46 & 47 & 48 & 49 & 50\end{array}$

Time (msec)

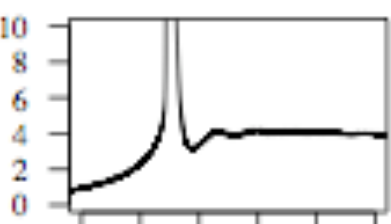

$\begin{array}{llllll}45 & 46 & 47 & 48 & 49 & 50\end{array}$

Time (msec)

Figure 3, Rebound cell dynamics

\section{Results}

Generally, we have been able to replicate in GENESIS simulations the firing patterns described in Sivaramakrishnan and Oliver [7]. Of some interest are the dynamics of the currents in rebound cells. Those cells respond to the end of hyperpolarizing current injection with a membrane potential rebound to values more depolarized than the resting potential of the cell. This 'rebound hump' is believed to be produced by the interaction of relatively slow voltage-activated $\mathrm{Ca}++$ channels with the $\mathrm{Na}+$ and $\mathrm{K}+$ Hodgkin-Huxley $(\mathrm{HH})$ channels that drive spike generation. Error! Reference source not found. illustrates the underlying conductances and the resulting membrane potentials in our modeling. In vivo the $\mathrm{Ca}++$ rebound appears to be a simple hump of depolarization with superimposed spikes, but the modeling suggests 
that it has a more complicated internal structure, involving the damping of a forced Hodgkin-Huxley system by Ca++ conductances.

\section{Discussion and Conclusions}

The rebound spike mechanism has been suggested as playing a role in measuring time delays between sounds [9], and the detailed interaction between channels that we have modeled with GENESIS may play a role in decreasing the variability of the timing of the rebound spike. Live animals seem to perform accurate measures of time delays between sounds-jitter sensitivity of 10-100 nanoseconds has been reported by Simmons, et al. [10] — and this channel mechanism may underlie auditory time delay hyperacuity.

Acknowledgments. This work was supported by the Engineering and Physical Sciences Research Council.

Address for reprint requests: $\mathrm{H}$ Erwin, University of Sunderland, School of Computing \& Technology, PO Box 299, Sunderland, SR6 0YN, United Kingdom

\section{References}

1. Rees, A., et al.: Regularity of Firing of Neurons in the Inferior Colliculus. Journal of Neurophysiology. 77 (1997) 2945-2965

2. Wilson, M., et al. GENESIS, the Caltech Neural Network Simulator. 1991 Available from: ftp://genesis.caltech.edu/

3. Wiener, M.C. and B.J. Richmond: Decoding Spike Trains Instant by Instant Using Order Statistics and the Mixture-of-Poissons Model. Journal of Neuroscience. 23 (2003) 2394-2406

4. Kass, R.E. and V. Ventura: A Spike-Train Probability Model. Neural Computation. 13 (2001) 1713-1720

5. Eden, U.T., et al.: Dynamic Analysis of Neural Encoding by Point Process Adaptive Filtering. Neural Computation. 16 (2004) 971-998

6. Brown, E.N., et al.: The Time-Rescaling Theorem and Its Application to Neural Spike Train Data Analysis. Neural Computation. 14 (2001) 325-341

7. Sivaramakrishnan, S. and D.L. Oliver: Distinct K Currents Result in Physiologically Distinct Cell Types in the Inferior Colliculus of Rat. Journal of Neuroscience. 21 (2001) 2861-2877

8. De Schutter, E. and J.M. Bower: An active membrane model of the cerebellar Purkinje cell: I. Simulation of current clamps in slice. Journal of Neurophysiology. 71 (1994) 375-400

9. Olsen, J.F.: Processing of biosonar information by the medial geniculate body of the mustached bat, Pteronotus parnelli. Washington University (1986)

10. Simmons, J.A., et al.: Discrimination of jittered sonar echoes by the echolocating bat, Eptesicus fuscus: the shape of target images in echolocation. Journal of Comparative Physiology A. 167 (1990) 589-616 
